\title{
COLORFUL LINEAR PROGRAMMING, NASH EQUILIBRIUM, AND PIVOTS
}

\author{
FRÉDÉRIC MEUNIER AND PAULINE SARRABEZOLLES
}

\begin{abstract}
The colorful Carathéodory theorem, proved by Bárány in 1982, states that given $d+1$ sets of points $\mathbf{S}_{1}, \ldots, \mathbf{S}_{d+1}$ in $\mathbb{R}^{d}$, with each $\mathbf{S}_{i}$ containing $\mathbf{0}$ in its convex hull, there exists a set $T \subseteq \bigcup_{i=1}^{d+1} \mathbf{S}_{i}$ containing $\mathbf{0}$ in its convex hull and such that $\left|T \cap \mathbf{S}_{i}\right| \leq 1$ for all $i \in\{1, \ldots, d+1\}$. An intriguing question - still open - is whether such a set $T$, whose existence is ensured, can be found in polynomial time. In 1997, Bárány and Onn defined colorful linear programming as algorithmic questions related to the colorful Carathéodory theorem. The question we just mentioned comes under colorful linear programming.

The traditional applications of colorful linear programming lie in discrete geometry. In this paper, we study its relations with other areas, such as game theory, operations research, and combinatorics. Regarding game theory, we prove that computing a Nash equilibrium in a bimatrix game is a colorful linear programming problem. We also formulate an optimization problem for colorful linear programming and show that as for usual linear programming, deciding and optimizing are computationally equivalent. We discuss then a colorful version of Dantzig's diet problem. We also propose a variant of the Bárány algorithm, which is an algorithm computing a set $T$ whose existence is ensured by the colorful Carathéodory theorem. Our algorithm makes a clear connection with the simplex algorithm and we discuss its computational efficiency. Related complexity and combinatorial results are also provided.
\end{abstract}

\section{INTRODUCTION}

1.1. Context. In 1982, Bárány proved a colorful generalization of the Carathéodory theorem, whose statement is the following.

Theorem 1 (Colorful Carathéodory theorem [2]). Given $d+1$ sets of points $\mathbf{S}_{1}, \ldots, \mathbf{S}_{d+1}$ in $\mathbb{R}^{d}$ such that each $\mathbf{S}_{i}$ contains $\mathbf{0}$ in its convex hull, there exists a set $T$ of the form $\left\{s_{1}, \ldots, s_{d+1}\right\}$, with $s_{i} \in \mathbf{S}_{i}$ for every $i \in\{1, \ldots, d+1\}$, that contains $\mathbf{0}$ in its convex hull.

A natural question raised by this theorem is whether such a colorful set $T$ can be computed in polynomial time. The case with $\mathbf{S}_{1}=\cdots=\mathbf{S}_{d+1}$, corresponding to the usual Carathéodory theorem, is known to be solvable in polynomial time, via linear programming. However, the complexity of the colorful version remains an open question.

In 1997, Bárány and Onn defined algorithmic and complexity problems related to the colorful Carathéodory theorem [4, giving birth to colorful linear programming. In their paper, the complexity question raised by the colorful Carathéodory theorem is referred as an "outstanding problem on the borderline of tractable and intractable problems". In addition of providing a theoretical challenge, the colorful Carathéodory theorem has several applications

Key words and phrases. Bimatrix games; colorful Carathéodory theorem; colorful linear programming; complexity; pivoting algorithms; Sperner's lemma. 
in discrete geometry (e.g. Tverberg partition, "first selection lemma", see [22]). Any efficient algorithm computing such a colorful set $T$ would benefit these applications.

A set of points is positively dependent if it is nonempty and contains $\mathbf{0}$ in its convex hull. Given a configuration of $k$ sets of points $\mathbf{S}_{1}, \ldots, \mathbf{S}_{k}$ in $\mathbb{R}^{d}$, a set $T$ is colorful if it is of the form $\left\{s_{1}, \ldots, s_{k}\right\}$ with $s_{i} \in \mathbf{S}_{i}$ for every $i \in\{1, \ldots, k\}$. One of the problems studied in this paper is the following.

\section{Colorful Carathéodory}

Input. A configuration of $d+1$ positively dependent sets of points $\mathbf{S}_{1}, \ldots, \mathbf{S}_{d+1}$ in $\mathbb{Q}^{d}$.

Task. Find a positively dependent colorful set.

As we have already mentioned, the complexity status is still open. A more general problem has been recently proved to be PLS-complete by Mulzer and Stein [26]. The PLS class, where PLS stands for "Polynomial Local Search", contains the problems for which local optimality can be verified in polynomial time [15]. The original proof of the colorful Carathéodory theorem by Bárány naturally provides an algorithm solving Colorful CARATHÉOdory. This algorithm, known as the Bárány algorithm, was analyzed and improved by Bárány and Onn [4. It is a pivot algorithm roughly relying on computing the closest facet of a simplex to 0. Although not polynomial, this algorithm is quite efficient, as proved by Deza et al. through an extensive computational study [10]. In addition to Colorful Carathéodory, Bárány and Onn formulated the following problem, which is in a sense more general.

\section{Colorful Linear Programming}

Input. A configuration of $k$ sets of points $\mathbf{S}_{1}, \ldots, \mathbf{S}_{k}$ in $\mathbb{Q}^{d}$.

Task. Decide whether there exists a positively dependent colorful set.

We emphasize that when we write "Colorful Linear Programming", with small capital letters, we refer to that problem, as in Bárány-Onn paper [4, but when we write "colorful linear programming", we mean the study of the family of all problems about finding or deciding the existence of a positively dependent colorful set.

Bárány and Onn showed that the case of Colorful Linear Programming with $k=d$ is NP-complete even if each $\mathbf{S}_{i}$ is of size 2, proving that the general case is NP-complete as well. It contrasts with COLORful CARAThÉodory. In this version, when each $\mathbf{S}_{i}$ is of size 2 , we clearly have a polynomial special case: select one point in each $\mathbf{S}_{i}$, find the linear dependency, and change for the other point in $\mathbf{S}_{i}$ for those having a negative coefficient.

A slightly more general version of COLORFul CARATHÉODORY can be defined with conic hulls instead of convex hulls.

COLORFul CARAThÉOdory (conic version)

Input. A configuration of $d$ sets of points $\mathbf{S}_{1}, \ldots, \mathbf{S}_{d}$ in $\mathbb{Q}^{d}$ and an additional point $\boldsymbol{p}$ in $\bigcap_{i=1}^{d} \operatorname{cone}\left(S_{i}\right) \cap \mathbb{Q}^{d}$.

Task. Find a colorful set $T$ such that $\boldsymbol{p} \in \operatorname{cone}(T)$. 
The colorful set $T$ exists for sure because of a conic version of the colorful Carathéodory theorem, also proved by Bárány in the same paper [2]. By an easy geometric argument, this

problem coincides with COLORFul CARAThÉOdory when conv $\left(\bigcup_{i=1}^{d} \mathbf{S}_{i}\right)$ does not contain 0. Note that as usual for this kind of problems, there is a shift in the dimension when going from one version to the other.

We define in the same way a conic version of Colorful Linear Programming.

Colorful Linear Programming (conic version)

Input. A configuration of $k$ sets of points $\mathbf{S}_{1}, \ldots, \mathbf{S}_{k}$ in $\mathbb{Q}^{d}$ and an additional point $\boldsymbol{p}$ in $\mathbb{Q}^{d}$. Task. Decide whether there exists a colorful set $T$ such that $\boldsymbol{p} \in \operatorname{cone}(T)$.

\subsection{Main contributions. "BIMATRIX is a colorful linear programming problem".}

We prove that the problem BIMATRIX, consisting in computing a Nash equilibrium in a bimatrix game, is polynomially reducible to Finding Another Colorful Simplex ProbLEM, which is a colorful linear programming problem introduced by Meunier and Deza [25]. It shows that this latter problem is PPAD-complete. This was stated as an open question in the cited paper. On our way, we introduce a new method for proving that a linear complementarity problem belongs to the PPAD class, based on Sperner's lemma. This method seems to be interesting for its own sake, since it avoids the introduction of oriented primoids, as done usually. All these results are stated and proved in Section 2, where a definition of the PPAD class is also provided.

\section{"The simplex algorithm solves COLORful CARAThÉOdory".}

In Section 3, we show that the Bárány algorithm and its improvement by Bárány and Onn can be slightly modified in order to get what is more or less a "Phase I" simplex method. Instead of computing a closest facet at each pivot step, we select a new point by a classical reduced cost consideration. It simplifies the iteration, and improves the complexity of this latter. A complexity analysis of the overall algorithm is provided, as well as numerical experiments that show the effectiveness of the approach.

\section{"Optimization and decision are equivalent for colorful linear programming".}

For the usual linear programming, it is known that the problem of deciding the existence of a solution to a linear program and the problem of optimizing a linear program are polynomially equivalent, see for instance Theorem 10.4 of Schrijver [30]. In Section 4, we show that a similar equivalence holds for colorful linear programming. We present then an operations research type application of this result, namely a colored version of the famous diet problem by Dantzig. We are not aware of similar applications in the literature, with industrial problems explicitly formulated as colorful linear programs.

In addition to these results, we show that Colorful Linear Programming is NPcomplete even if $k-d$ is fixed and each $\mathbf{S}_{i}$ is of size 2 . The proof is easy and this result should rather be called an observation. However, the complexity status of the case $k-d=1$ was a question of Bárány and Onn (Section 5 of [4]) and it seems that people are not aware 
of the fact that the answer is easy. As a by-product of this complexity result, we get a new proof of the coNP-completeness of deciding whether a polytope is the projection of another polytope, both being described by systems of linear inequalities. These results are stated and proved in Section 5 .

We end the paper with a study of special cases and analogues of colorful linear programming in combinatorics (Section 6). In particular, two combinatorial and polynomial cases of Colorful Carathéodory are presented. They are rather easy, but are not stated elsewhere and are clearly relevant for the understanding of the colorful linear programming landscape.

\section{Links With NASH EQUiLibRia}

2.1. Another problem. The fact that computing Nash equilibria is a colorful linear programming problem relies on the study of another problem similar to ColORFUl CARATHÉOdorY. This problem was proposed by Meunier and Deza [25] as a byproduct of an existence theorem, the Octahedron lemma [3, 9], which by some features has a common flavor with the colorful Carathéodory theorem. The Octahedron lemma states that if each $\mathbf{S}_{i}$ of the configuration is of size 2 and if the points are in general position, the number of positively dependent colorful sets is even. By general position, we mean that no $d^{\prime}+1$ points of the input points, $\mathbf{0}$ included, lie in a same $\left(d^{\prime}-1\right)$-dimensional affine subspace.

The problem we call Finding Another Colorful Simplex is the following.

Finding Another Colorful Simplex

Input. A configuration of $d+1$ pairs of points $\mathbf{S}_{1}, \ldots, \mathbf{S}_{d+1}$ in $\mathbb{Q}^{d}$ and a positively dependent colorful set in this configuration.

Task. Find another positively dependent colorful set.

Another positively dependent colorful set exists for sure. Indeed, by a slight perturbation, we can assume that all points are in general position. If there were only one positively dependent colorful set, there would also be only one positively dependent colorful set in the perturbed configuration, which violates the evenness property stated by the Octahedron lemma. In their paper, Meunier and Deza question the complexity status of this problem. We solve the question by proving that it is actually a generalization of the problem of computing a Nash equilibrium in a bimatrix game.

2.2. Finding Another Colorful Simplex is in PPAD. In [25], it was noted that FindIng Another Colorful Simplex is in PPA. The class PPA, also defined by Papadimitriou in 1994 [27], contains the class PPAD. PPA contains the problems that can be polynomially reduced to the problem of finding another degree 1 vertex in a graph whose vertices all have degree at most 2 and in which a degree 1 vertex is already given. The graph is supposed to be implicitly described by the neighborhood function, which, given a vertex, returns its neighbors in polynomial time. The PPAD class is the subclass of PPA for which the implicit graph is oriented and such that each vertex has an outdegree at most 1 and an indegree at most 1 . The problem becomes then: given an unbalanced vertex, that is a vertex 
$v$ such that $\operatorname{deg}^{+}(v)+\operatorname{deg}^{-}(v)=1$, find another unbalanced vertex. See [27] for further precisions.

We prove in this subsection that Finding Another Colorful Simplex is in PPAD. We proceed by showing that the existence of the other positively dependent colorful set is a consequence of Sperner's lemma 32. Our method for proving that Finding Another Colorful Simplex belongs to PPAD is adaptable for other complementarity problems, among them Bimatrix. We believe that our method is new. It avoids the use, as in [27, 6, 16, 35, of oriented primoids or oriented duoids defined by Todd [34].

One of the multiple versions of Sperner's lemma is the following theorem, proposed by Scarf [29], which involves a triangulation of a sphere whose vertices are labeled. A simplex whose vertices get pairwise distinct labels is fully-labeled.

Theorem 2 (Sperner's lemma). Let $\mathrm{T}$ be a triangulation of an $n$-dimensional sphere $\mathcal{S}^{n}$ and let $V$ be its vertex set. Assume that the elements of $V$ are labeled according to a map $\lambda: V \rightarrow E$, where $E$ is some finite set. If $E$ is of cardinality $n+1$, then there is an even number of fully-labeled n-simplices.

We state now the main proposition of this subsection.

Proposition 1. Finding Another Colorful Simplex is in PPAD.

The proof uses the following fact. It is an elementary fact about feasible bases in linear programming, but since we were not able to find any place where it is clearly stated and proved, we do it for sake of completeness. Let us consider the system of linear equalities and inequalities of the form

$$
A \boldsymbol{x}=\boldsymbol{b}, \boldsymbol{x} \geq \mathbf{0},
$$

where $A$ is an $m \times n$ real matrix of rank $m$ and let $\boldsymbol{b} \in \mathbb{R}^{m}$. This system is nondegenerate if any feasible solution has a support of size at least $m$.

Lemma 1 ("Complements of feasible bases form a sphere"). Let $P$ be the set of feasible solutions of $(\mathrm{S})$. Suppose that $(\mathrm{S})$ is nondegenerate and that $P$ is bounded. Then $\{\{1, \ldots, n\} \backslash \operatorname{supp}(\boldsymbol{x}): \boldsymbol{x} \in P\}$ is a simplicial complex that is isomorphic to a triangulation of an $(n-m-1)$-dimensional sphere $\mathcal{S}^{n-m-1}$.

Proof. Denote by $\mathrm{K}$ the simplicial complex $\{\{1, \ldots, n\} \backslash \operatorname{supp}(\boldsymbol{x}): \boldsymbol{x} \in P\}$. It is a simplicial complex because the system is nondegenerate. The vertices of $\mathrm{K}$ are in one-to-one correspondence with the facets of $P$, which are in one-to-one correspondence with the vertices of the polar $P^{*}$ of $P$. The resulting one-to-one correspondence between the vertices of $\mathrm{K}$ and the vertices of $P^{*}$ leads to a simplicial map between $\mathrm{K}$ and the face complex of $\partial P^{*}$ (here we use the correspondence between the faces of $P$ and $P^{*}$ as stated in Proposition 5.3.5 of [22]). The inverse of this map is also simplicial. Thus $\mathrm{K}$ is isomorphic to the face complex of $\partial P^{*}$.

Proof of Proposition 11. Define $A$ as the $(d+1) \times(2 d+2)$ matrix, whose columns are the coordinates of the input points, with an additional 1 at the $(d+1)$ th position. This is done "with repetitions" and the columns are numbered so that the points in $\mathbf{S}_{i}$ correspond to columns $2 i-1$ and $2 i$ (thus, if a point is in several $\mathbf{S}_{i}$ 's, it will provide several columns). 
Define $\boldsymbol{b}$ as the vector with 0 at every position except at the $(d+1)$ th position, where we also have a 1. By a perturbation argument - see [23] for instance for a description of such a polynomial-time computable perturbation - we can assume that the system

$$
A \boldsymbol{x}=\boldsymbol{b}, \boldsymbol{x} \geq \mathbf{0}
$$

is nondegenerate. Every feasible basis of this system that contains exactly one element of each $\{2 i-1,2 i\}$ - which we call a colorful feasible basis - provides a positively dependent colorful set. We describe now a polynomial reduction of the problem of finding a colorful basis in this system to the problem of finding a fully-labeled simplex in a triangulation of a $d$-dimensional sphere.

We define a simplicial complex $\mathrm{K}$ with vertex set $\{1, \ldots, 2 d+2\}$ and whose simplices $\sigma$ are such that $\{1, \ldots, 2 d+2\} \backslash \sigma$ is the support of a feasible solution of the system. $\mathrm{K}$ is a simplicial complex of the form stated in Lemma 1. It is thus a triangulation of $\mathcal{S}^{d}$, with $m=d+1$ and $n=2 d+2$. Now, for $v$ a vertex of $\mathrm{K}$, define $\lambda(v)$ to be its color, i.e., the index $i$ such that $v \in\{2 i-1,2 i\}$. Any fully-labeled simplex $\sigma$ of $\mathrm{K}$ is such that $\{1, \ldots, 2 d+2\} \backslash \sigma$ is a colorful feasible basis and conversely. There is thus an explicit one-to-one correspondence between the fully-labeled simplices of $\mathrm{K}$ and the colorful feasible bases. The triangulation $\mathrm{K}$ here can easily be encoded by a Turing machine computing the neighbors of any simplex in the triangulation in polynomial time. There is a proof of Sperner's lemma (Theorem 21) via an oriented path-following argument [29, 24, which considers directed paths joining fullylabeled simplices. Given a fully-labeled simplex, finding another fully-labeled simplex is thus in PPAD, and so is Finding Another Colorful Simplex.

2.3. Reduction of Bimatrix. A bimatrix game involves two $m \times n$ matrices with real coefficients $A=\left(a_{i j}\right)$ and $B=\left(b_{i j}\right)$. There are two players. The first player chooses a probability distribution on $\{1, \ldots, m\}$, the second a probability distribution on $\{1, \ldots, n\}$. Once these probability distributions have been chosen, a pair $(\bar{i}, \bar{j})$ is drawn at random according to these distributions. The first player gets a payoff equal to $a_{(\bar{i}, \bar{j})}$ and the second a payoff equal to $b_{(\bar{i}, \bar{j})}$. A Nash equilibrium is a choice of distributions in such a way that if a player changes his distribution, he will not get in average a strictly better payoff.

Let $\Delta^{k}$ be the set of vectors $\boldsymbol{x} \in \mathbb{R}_{+}^{k}$ such that $\sum_{i=1}^{k} x_{i}=1$. Formally, a Nash equilibrium is a pair $\left(\boldsymbol{y}^{*}, \boldsymbol{z}^{*}\right)$ with $\boldsymbol{y}^{*} \in \Delta^{m}$ and $\boldsymbol{z}^{*} \in \Delta^{n}$ such that

$$
\boldsymbol{y}^{\prime T} A \boldsymbol{z}^{*} \leq \boldsymbol{y}^{* T} A \boldsymbol{z}^{*} \text { for all } \boldsymbol{y}^{\prime} \in \Delta^{m} \quad \text { and } \quad \boldsymbol{y}^{* T} B \boldsymbol{z}^{\prime} \leq \boldsymbol{y}^{* T} B \boldsymbol{z}^{*} \text { for all } \boldsymbol{z}^{\prime} \in \Delta^{n} .
$$

It is well-known that if the matrices have rational coefficients, there is a Nash equilibrium with rational coefficients, which are not too large with respect to the input. Bimatrix is the following problem: given $A$ and $B$ with rational coefficients, find a Nash equilibrium. Papadimitriou showed in 1994 that Bimatrix is in PPAD [27]. Later, Chen, Deng, and Teng [6] proved its PPAD-completeness.

A combinatorial approach to these equilibria consists in studying the complementary solutions of the two systems

$$
\left[A, I_{m}\right] \boldsymbol{x}=(1, \ldots, 1)^{T} \text { and } \boldsymbol{x} \in \mathbb{R}_{+}^{n+m}
$$

and

$$
\left[I_{n}, B^{T}\right] \boldsymbol{x}=(1, \ldots, 1)^{T} \text { and } \boldsymbol{x} \in \mathbb{R}_{+}^{n+m}
$$


By complementary solutions, we mean a solution $\boldsymbol{x}_{A}$ of (2) and a solution $\boldsymbol{x}_{B}$ of (3) such that $\boldsymbol{x}_{A} \cdot \boldsymbol{x}_{B}=0$. Indeed, complementary solutions with $\operatorname{supp}\left(\boldsymbol{x}_{A}\right) \neq\{n+1, \ldots, n+m\}$ or $\operatorname{supp}\left(\boldsymbol{x}_{B}\right) \neq\{1, \ldots, n\}$ give a Nash equilibrium. This point of view goes back to Lemke and Howson [20]. A complete proof within this framework can be found in Remark 6.1 of [24].

We derive the difficulty of Finding Another Colorful Simplex from the complexity of BIMATRIX.

\section{Proposition 2. Finding Another Colorful Simplex is PPAD-complete.}

Proof. We prove that the following version of Finding Another Colorful Simplex with cones is PPAD-complete. This version is equivalent to Finding Another Colorful SIMPLEX.

\section{Finding Another Colorful Cone}

Input. A configuration of $d+1$ pairs of points $\mathbf{S}_{1}, \ldots, \mathbf{S}_{d+1}$ in $\mathbb{Q}^{d+1}$, an additional point $\boldsymbol{p}$ in $\mathbb{Q}^{d+1}$ such that $\operatorname{conv}\left(\bigcup_{i=1}^{d+1} \mathbf{S}_{i}\right)$ does not contain $\mathbf{0}$, and a colorful set $T$ such that $\boldsymbol{p} \in \operatorname{cone}(T)$. Task. Find another colorful set $T^{\prime}$ such that $\boldsymbol{p} \in \operatorname{cone}\left(T^{\prime}\right)$.

The proof uses a reduction of Bimatrix to Finding Another Colorful Cone. Consider an instance of BIMATRIX. First note that we can assume that all coefficients of $A$ and $B$ are positive. Indeed, adding a same constant to all entries of the matrices does not change the game. Build the $(m+n) \times(2(m+n))$ matrix

$$
M=\left(\begin{array}{cccc}
A & I_{m} & \mathbf{0} & \mathbf{0} \\
\mathbf{0} & \mathbf{0} & I_{n} & B^{T}
\end{array}\right) .
$$

We denote by $M_{i}$ the $i$ th column of $M$. Note that the vector $\boldsymbol{u}=(1, \ldots, 1) \in \mathbb{R}^{n+m}$ is in the conic hull of $T=\left\{M_{n+1}, \ldots, M_{n+m}, M_{n+m+1}, \ldots, M_{2 n+m}\right\}$. Indeed, the corresponding submatrix is the identity matrix.

Let $\mathbf{S}_{i}$ be the pair $\left\{M_{i}, M_{m+n+i}\right\}$ for $i=1, \ldots, m+n$. Since all coefficients of $A$ and $B$ are positive, $\mathbf{0}$ is not in the convex hull of the columns of $M$ and $\boldsymbol{u}$. A polynomial time algorithm solving Finding Another Colorful Simplex with $T$ as input set would find another colorful set $T^{\prime}$ such that $\boldsymbol{u} \in \operatorname{cone}\left(T^{\prime}\right)$. The decomposition of $\boldsymbol{u}$ on the points in $T^{\prime}$ gives a nonnegative vector $\boldsymbol{x}$ such that $M \boldsymbol{x}=\boldsymbol{u}, x_{i} x_{m+n+i}=0$ for $i=1, \ldots, m+n$, and $\operatorname{supp}(\boldsymbol{x}) \neq$ $\{n+1, \ldots, 2 n+m\}$. Such an $\boldsymbol{x}$ can be written $\left(\boldsymbol{x}_{A}, \boldsymbol{x}_{B}\right)$ with $\boldsymbol{x}_{A}, \boldsymbol{x}_{B} \in \mathbb{R}_{+}^{m+n}$ satisfying $\boldsymbol{x}_{A} \cdot \boldsymbol{x}_{B}=0$ and such that $\operatorname{supp}\left(\boldsymbol{x}_{A}\right) \neq\{n+1, \ldots, n+m\}$ or $\operatorname{supp}\left(\boldsymbol{x}_{B}\right) \neq\{1, \ldots, n\}$. In other words, it would find a Nash equilibrium. Bimatrix being PPAD-complete, Proposition 1 implies therefore that Finding Another Colorful Simplex is PPAD-complete.

This proof shows that Finding Another Colorful Simplex is more general than computing complementary solutions of Equations (22) and (3). In [25], a pivoting algorithm for solving Finding Another Colorful Simplex is proposed. It reduces to the classical pivoting algorithm due to Lemke and Howson [20] used for computing such complementary solutions.

Remark 1. A reviewer noted that Propositions 1 and 2 are close to results presented in a paper by Király and Pap [17]. Actually, using results of the latter paper, we can directly prove Proposition 1. As suggested by the referee, consider the set of all $(2 d+2)$-dimensional 
vectors corresponding to coefficients of positively dependences. It is a $(d+1)$-dimensional polytope $Q$ with exactly $(2 d+2)$ facets, each facet corresponding to a point from the input with a 0 coefficient in the positive dependence. Color each facet with the color of the corresponding input point. The positively dependent colorful sets correspond to vertices of $Q$ surrounded by facets of distinct colors ("panchromatic vertices"). The Theorem 2 of their paper ensures that if there is a panchromatic vertex, there is another one, thus showing the Octahedron lemma, and the related computational problem is implicitly proved to be PPAD. We get thus Proposition 1 by this way. We did not find a direct and easy way for proving Proposition 2 with the results of the mentioned paper, but it should be possible.

However, we think that the proof we propose for Proposition 1 remains interesting, especially because it describes a systematic way for proving PPAD-membership of linear complementary problems. Moreover, it reveals that an elementary Sperner-type computational problem is PPAD-complete, see next remark.

Remark 2 (Complexity of Sperner's lemma). Consider the problem

Input. A triangulation $\mathrm{T}$ of $\mathcal{S}^{d}$ involving $2(d+1)$ vertices, a labeling $\lambda: V(\mathrm{~T}) \rightarrow$ $\{1, \ldots, d+1\}$, and a fully-labeled simplex.

Task. Find another fully-labeled simplex.

The reduction used in the proof of Proposition 1 and Proposition 2 shows that this problem is actually PPAD-complete, even if each label appears exactly twice. Sperner-type problems have already been proved to be PPAD-complete [5, 27], but these latter problems are in fixed dimension, with an exponential number of vertices, and with a labeling given by an oracle, while the Sperner-type problem we introduce has an explicit description of the vertices and of the labeling. Note that the number of vertices is small. As already mentioned, the paper by Király and Pap [17] present and discuss computational problems that are in the same spirit and also proved to be PPAD-complete, but they are presented in a form that makes them farther from the classical Sperner's lemma: their formulation involves an unbounded polyhedron, with a coloring of the facets, and some conditions on the extreme rays.

Remark 4 in Section [6 will exhibit some polynomial cases of the Sperner-type problem we introduce here.

\section{SimplexificAtion OF BÁRÁNY's ALGORITHM}

The proof of the colorful Carathéodory theorem by Bárány [2] actually provides an algorithm. This algorithm was improved and analyzed by Bárány and Onn in 1997. In this section, we propose a new algorithm to compute a colorful solution. It can be seen as a "simplexification" of Bárány's algorithm. This latter requires at each iteration to compute the projection of the origin on a simplex. We show that this projection operation, which is not an easy task, although polynomial, can be replaced by a simple reduced cost test. This leads to an algorithm that is almost the classical simplex algorithm.

3.1. Bárány's algorithm. The pivoting algorithm proposed by Bárány for solving CoLorful Carathéodory goes as follows. The input is the sets $\mathbf{S}_{1}, \ldots, \mathbf{S}_{d+1}$ of points in $\mathbb{Q}^{d}$, 
each of cardinality $d+1$ and positively dependent.

\section{Bárány's algorithm}

- Choose a first colorful set $T_{1}$ of size $d+1$, let $i:=0$, and let $\boldsymbol{x}_{1}$ be the point of minimum norm in $\operatorname{conv}\left(T_{1}\right)$.

- Repeat:

- Let $i:=i+1$.

- If $\mathbf{0} \in \operatorname{conv}\left(T_{i}\right)$, stop and output $T_{i}$.

- Otherwise, find $\boldsymbol{x}_{i}$ of minimum norm in $\operatorname{conv}\left(T_{i}\right)$; choose $\boldsymbol{t}_{i} \in T_{i}$ such that $\boldsymbol{x}_{i} \in \operatorname{conv}\left(T_{i} \backslash\left\{\boldsymbol{t}_{i}\right\}\right)$; choose a point $\boldsymbol{t}$ in $\mathbf{S}_{k}$ minimizing $\boldsymbol{t} \cdot \boldsymbol{x}_{i}$, where $k$ is the color of $\boldsymbol{t}_{i}$; define $T_{i+1}:=T_{i} \backslash\left\{\boldsymbol{t}_{i}\right\} \cup\{\boldsymbol{t}\}$.

It is rather straightforward to prove that the sequence $\left(\left\|\boldsymbol{x}_{i}\right\|\right)$ converges to 0 when $i$ goes to infinity, see [4] for the details. By finiteness, it implies that Bárány's algorithm finds a positively dependent colorful set in finite time. The technical step is the computation of $\boldsymbol{x}_{i}$, which is a projection computation. It is a rather heavy task, which can moreover only be solved approximately. The improvement of Bárány and Onn consists in replacing this projection by the computation of another point that plays a similar role, but which requires only linear algebra for its computation. Deza et al. [10] shows how to compute this point in $O\left(d^{3}\right)$. They also proceed to an extensive experimental study of algorithms solving this problem. In addition to some heuristics, "multi-update" versions are also proposed, but they do not avoid this kind of operations.

Our algorithm, described in the next section, allows to do each iteration in $O\left(d^{2}\right)$. Perhaps more interestingly, it shows that in some sense the simplex algorithm solves COLORFUL CARATHÉODORY, with almost all classical pivot-rules as possible variations.

\subsection{The simplex version.}

3.2.1. The algorithm. We add a dummy point $\boldsymbol{v}$ and define the following optimization problem.

$$
\begin{array}{ll}
\min & z \\
\text { s.t. } & \left(\begin{array}{ccc} 
& M & \\
1 & \ldots & 1
\end{array}\right) \boldsymbol{\lambda}+z \overline{\boldsymbol{v}}=\left(\begin{array}{c}
0 \\
\vdots \\
0 \\
1
\end{array}\right) \\
& \boldsymbol{\lambda} \geq \mathbf{0}, z \geq 0,
\end{array}
$$

where $\overline{\boldsymbol{v}}=(\boldsymbol{v}, 1)$ and where $M$ is the $d \times(d+1)^{2}$ matrix whose columns are the points of $\bigcup_{i=1}^{d+1} \mathbf{S}_{i}$ (where as in the proof of Proposition 1 we consider the disjoint union of the points). This optimization problem simply looks for an expression of $\mathbf{0}$ as a convex combination of the points in $\{\boldsymbol{v}\} \cup \bigcup_{i=1}^{d+1} \mathbf{S}_{i}$ with a minimal weight on $\boldsymbol{v}$. Especially, if $\mathbf{0} \in \operatorname{conv}\left(\bigcup_{i} \mathbf{S}_{i}\right)$, the optimal value is 0 . The idea consists in seeking an optimal basis, with the terminology of linear programming, which in addition is required to be colorful. The colorful Carathéodory theorem ensures that such a basis exists. 
Now, choose a first transversal $F_{1}$, which is a colorful set of cardinality $d$. Choose the dummy point $\boldsymbol{v}$ so that $F_{1} \cup\{\boldsymbol{v}\}$ contains $\mathbf{0}$ in the interior of its convex hull (we explain below in Section 3.2 .2 how it is possible to assume the existence of such set and point, and how to compute them). Note that $F_{1} \cup\{\boldsymbol{v}\}$ is a feasible basis. The algorithm proceeds with simplex pivots, going from feasible colorful basis to feasible colorful basis, until an optimal colorful basis is found. We start with $i:=0$. We repeat then

- Let $i:=i+1$.

- Choose a point $\boldsymbol{t}$ of the missing color in $F_{i}$ with negative reduced cost. The reduced costs are computed according to the current basis $F_{i} \cup\{\boldsymbol{v}\}$.

- Proceed to a simplex pivot operation with $\boldsymbol{t}$ entering the current basis.

- If $\boldsymbol{v}$ leaves the basis, stop and output $F_{i} \cup\{\boldsymbol{t}\}$ (it is an optimal colorful basis).

- Otherwise, define $F_{i+1}$ to be the new basis minus $\boldsymbol{v}$.

The following lemma shows that as long as a positively dependent colorful set has not been found, selecting a point of a color missed by $F_{i}$ with a negative reduced cost is the same as selecting a point in the open half-space delimited by aff $\left(F_{i}\right)$ and containing $\mathbf{0}$. As in Bárány's algorithm, we get closer and closer to the origin, and by finiteness, we eventually find a positively dependent colorful set.

Lemma 2. The points in the open half-space delimited by aff $\left(F_{i}\right)$ and containing $\mathbf{0}$ are precisely the points with a negative reduced cost.

Proof. Let $F_{i}=\left\{\boldsymbol{u}_{1}, \ldots, \boldsymbol{u}_{d}\right\}$ and let $\boldsymbol{t}$ be any other point in $\left(\bigcup_{j=1}^{d+1} \mathbf{S}_{j}\right) \backslash F_{i}$. Consider $x_{1}, \ldots, x_{d}, r, s \in \mathbb{R}$ such that

$$
\boldsymbol{t}+s \boldsymbol{v}+\sum_{i=1}^{d} x_{i} \boldsymbol{u}_{i}=\mathbf{0} \quad \text { and } \quad 1+s+\sum_{i=1}^{d} x_{i}=0
$$

The reduced cost of $\boldsymbol{t}$ is exactly $s$. Therefore, proving the lemma amounts to prove that $s$ is negative exactly when $\boldsymbol{t}$ is in the open half-space delimited by aff $\left(F_{i}\right)$ and containing $\mathbf{0}$. To see this, note that (4) implies

$$
\left(\boldsymbol{t}-\boldsymbol{u}_{1}\right)+s\left(\boldsymbol{v}-\boldsymbol{u}_{1}\right)+\sum_{i=2}^{d} x_{i}\left(\boldsymbol{u}_{i}-\boldsymbol{u}_{1}\right)=\mathbf{0} .
$$

Now, choose a unit vector $\boldsymbol{n}$ orthogonal to aff $\left(F_{i}\right)$. Take the scalar product of Equation (5) and $\boldsymbol{n}$. It gives

$$
r \boldsymbol{n} \cdot\left(\boldsymbol{t}-\boldsymbol{u}_{1}\right)+s \boldsymbol{n} \cdot\left(\boldsymbol{v}-\boldsymbol{u}_{1}\right)=0
$$

and the conclusion follows since $\boldsymbol{v}$ and $\mathbf{0}$ are in the same half-space delimited by aff $\left(F_{i}\right)$.

This approach is reminiscent of the "Phase I" simplex method, which computes a first feasible basis by solving an auxiliary linear program whose optimal value is 0 on such a basis. 
3.2.2. Preprocessing. Computation of $F_{1}$. The algorithm requires to have a first transversal $F_{1}$ whose elements are linearly independent. If there is no such transversal, it means by Rado's theorem ([28], see also p.702 of [31]) that there exists $I \subseteq[d]$ such that $\operatorname{rank}\left(\bigcup_{i \in I} \mathbf{S}_{i}\right)<$ $|I|$. Deciding the existence of the transversal and computing $I$ if it does not exist can be done in polynomial time with the help of Edmonds' matroid intersection algorithm. Now, we remove all colors not in $I$ and consider COLORful CARATHÉODORY in the linear vector space spanned by $\bigcup_{i \in I} \mathbf{S}_{i}$. We can thus repeat this process several times if necessary and we will eventually get an input with a transversal whose elements are linearly independent.

Computation of $\boldsymbol{v}$. It is easy to choose $\boldsymbol{v}$. A way to do it that makes the complexity computation required for Proposition 3 below easy is to define $\boldsymbol{v}$ as $-\sum_{i=1}^{d} \boldsymbol{u}_{i}$, where the $\boldsymbol{u}_{i}$ 's are the vertices of $F_{1}$.

3.2.3. Complexity analysis. As for the classical simplex algorithm, it is not easy to provide a complexity analysis. We can however prove the following result.

Proposition 3. Suppose that the $\mathbf{S}_{i}$ 's and $\mathbf{0}$ are in general position. Suppose moreover that all points have integer coordinates. Then the algorithm computes a colorful set $T$ in at most $\left(d^{8}+1\right) \Delta^{4}$ iterations, where $\Delta$ is the largest absolute value of any $d \times d$ subdeterminant of the input points (i.e., of $M$ ).

Proof. Let us write the program ( $(\mathrm{P})$ under the form

$$
\begin{array}{ll}
\min & \boldsymbol{c} \cdot \boldsymbol{x} \\
\text { s.c. } & A \boldsymbol{x}=\boldsymbol{b} \\
& \boldsymbol{x} \geq \mathbf{0},
\end{array}
$$

where

$$
\boldsymbol{b}=\boldsymbol{c}=\left(\begin{array}{c}
0 \\
\vdots \\
0 \\
1
\end{array}\right) \quad \text { and } \quad A=\left(\begin{array}{cccc} 
& M & -\sum_{i=1}^{d} \boldsymbol{u}_{i} \\
1 & \ldots & 1 & 1
\end{array}\right)
$$

Let $\delta_{P}$ and $\gamma_{P}$ be respectively the minimum and maximum values of all positive elements of basic feasible solutions of the program $(\mathrm{P})$. For a feasible basis $B$, denote by $\boldsymbol{s}^{B}$ the corresponding reduced costs. Define then

$$
\delta_{D}^{\prime}=\min \left\{-s_{j}^{B}: B \text { is a feasible basis and } s_{j}^{B}<0\right\}
$$

and

$$
\gamma_{D}^{\prime}=\max \left\{-s_{j}^{B}: B \text { is a feasible basis and } s_{j}^{B}<0\right\} .
$$

A theorem by Kitahara and Minzuno [18] states that the number of different basic feasible solutions generated by the algorithm, and thus the number of pivot steps when the input is nondegenerate, is at most

$$
\left\lceil d \frac{\gamma_{P} \gamma_{D}^{\prime}}{\delta_{P} \delta_{D}^{\prime}}\right\rceil
$$

In order to apply this theorem, we bound $\delta_{P}, \gamma_{P}, \delta_{D}$, and $\gamma_{D}$. 
For a feasible basis $B$, the corresponding feasible basic solution is given by

$$
\boldsymbol{x}_{B}=A_{B}^{-1} \boldsymbol{b} \quad \text { and } \quad \boldsymbol{x}_{N}=\mathbf{0} .
$$

We have $A_{B}^{-1}=\frac{1}{\operatorname{det} A_{B}}\left(\text { comatrix } A_{B}\right)^{T}$. Note that the last column of $A$ is always in any feasible basis $B$ generated by the algorithm, otherwise we would be at the optimal solution of $(\mathrm{P})$. We compute $\operatorname{det}\left(A_{B}\right)$ by expanding along the last row. Since the last column of $A$ is the opposite of the sum of $d$ other columns, we get that $\operatorname{det}\left(A_{B}\right) \leq\left(d^{2}+1\right) \Delta$. Using that the input points have integer coordinates, we get

$$
\delta_{P} \geq \frac{1}{\left(d^{2}+1\right) \Delta}
$$

The input points having integer coordinates we have $\operatorname{det} A_{B} \geq 1$. Using Cramer's formula, we get

We have

$$
\gamma_{P} \leq d \Delta
$$

$$
\boldsymbol{s}_{B}^{B}=\mathbf{0} \quad \text { and } \quad \boldsymbol{s}_{N}^{B}=\boldsymbol{c}_{N}-A_{N}^{T}\left(A_{B}^{T}\right)^{-1} \boldsymbol{c}_{B} .
$$

Here, $\boldsymbol{c}_{N}=\mathbf{0}$ and $\boldsymbol{c}_{B}$ is the $d$ th standard unit vector, except for the final basis computed by the algorithm. Using exactly the same approach as for $\delta_{P}$ and $\gamma_{p}$ to bound the entries of $A_{N}^{T}\left(A_{B}^{T}\right)^{-1}$, we get that

$$
\delta_{D}^{\prime} \geq \frac{1}{\left(d^{2}+1\right) \Delta} \quad \text { and } \quad \gamma_{D}^{\prime} \leq\left(d^{2}+1\right) \Delta .
$$

We are not able to propose a convincing way to compare the theoretical complexity of Bárány-Onn's algorithm with ours. However, our algorithm works also for the conic version of Colorful CARAThÉodory, and in this case, our analysis provides the following result. The first transversal can be assumed to exist using a similar approach as in Section 3.2.2. by applying Rado's theorem, we can assume that there exists a linearly independent colorful set; the first transversal is given by any $(d-1)$-subset $F_{1}$ of this independent colorful set whose linear span does not contain $\boldsymbol{p}$.

Proposition 4. Consider COLORful CARAThÉodory in its conic version. Suppose that the matrix $M$ whose columns are the $d^{2}$ input points is totally unimodular and that $\boldsymbol{p}$ is in $\mathbb{Z}^{d}$. If the system

$$
M \boldsymbol{x}=\boldsymbol{p}, \boldsymbol{x} \geq \mathbf{0}
$$

is nondegenerate, then the algorithm computes a colorful cone containing $\boldsymbol{p}$ in at most $d^{6}\left(\|\boldsymbol{p}\|_{\infty}+1\right)^{4}$ pivot steps.

Proof. We define $\boldsymbol{v}$ to be $\boldsymbol{p}-\sum_{i=1}^{d-1} \boldsymbol{u}_{i}$, where the $\boldsymbol{u}_{i}$ 's form the subset $F_{1}$. We have then

$$
\delta_{P} \geq \frac{1}{d\left(\|\boldsymbol{p}\|_{\infty}+1\right)} \quad \text { and } \quad \gamma_{P} \leq d^{2}\|\boldsymbol{p}\|_{\infty}
$$

and

$$
\delta_{D}^{\prime} \geq \frac{1}{d\left(\|\boldsymbol{p}\|_{\infty}+1\right)} \quad \text { and } \quad \gamma_{D}^{\prime} \leq d\left(\|\boldsymbol{p}\|_{\infty}+1\right)
$$


3.2.4. Numerical results. We implemented our algorithm in $\mathrm{C}++$. The tests are performed on a PC Intel ${ }^{\circledR}$ Core $^{\mathrm{TM}}$ i3-2310M, with two 64-bit CPUs, clocked at $2.1 \mathrm{GHz}$, with 4 GB RAM. The instances are provided by five random generators, implemented by Huang in MATLAB and described in his master thesis [14]. All the generators provide instances of $(d+1)^{2}$ points in general position on the unit sphere, partitioned into $d+1$ colors and such that the origin $\mathbf{0}$ is in the convex hull of each color. Descriptions of the generators can be found in [13]. At each iteration, we choose the entering point $\boldsymbol{t}$ that has the most negative reduced cost.

Table 1 presents the computational results on 50 instances by dimension and by generators (the "tube" instances are those referred as "unbalanced" in Huang's master thesis). The columns "time" give the average execution time of the algorithm in milliseconds. The columns "\# pivots" give the average number of pivots. The entry corresponding to the "tube" case in dimension 384 is empty, since we faced cycling behavior for some instances (we felt that adding anti-cycling pivot rules was not imperative for our experiments).

\begin{tabular}{r|r|r|r|r|r|r|r|r|r|r} 
& \multicolumn{2}{|c|}{ Random } & \multicolumn{2}{|c|}{ Tube } & \multicolumn{2}{c|}{ Highdensity } & \multicolumn{2}{c|}{ Lowdensity } & \multicolumn{2}{c}{ Middensity } \\
Dimension & time & \# pivots & time & \# pivots & time & \# pivots & time & \# pivots & time & \# pivots \\
\hline 3 & 0.0135 & 1.94 & 0.0123 & 2.02 & 0.0342 & 1.62 & 0.0138 & 2.32 & 0.0170 & 1.70 \\
6 & 0.0180 & 3.38 & 0.0195 & 3.42 & 0.0474 & 1.98 & 0.0213 & 6.50 & 0.0164 & 2.88 \\
12 & 0.0406 & 6.56 & 0.0396 & 7.68 & 0.0609 & 1.84 & 0.0591 & 19.00 & 0.0371 & 4.88 \\
24 & 0.1433 & 13.76 & 0.1574 & 19.66 & 0.0871 & 1.94 & 0.2958 & 51.06 & 0.1123 & 9.62 \\
48 & 0.9612 & 31.86 & 1.1684 & 43.88 & 1.1006 & 1.94 & 2.7946 & 133.70 & 0.7725 & 19.44 \\
96 & 8.5069 & 76.42 & 11.2441 & 108.10 & 3.1116 & 1.92 & 28.5813 & 349.44 & 6.1306 & 39.46 \\
192 & 81.0170 & 186.62 & 250.1050 & 284.96 & 21.6753 & 1.86 & 263.1400 & 831.26 & 50.2998 & 93.88 \\
384 & 1111.5020 & 476.50 & & & 441.1310 & 2.00 & 5987.8880 & 2032.60 & 846.9148 & 279.12
\end{tabular}

TABLE 1. Average solution time and number of pivots for the simplex-like algorithm

We compare these results with those of the Bárány-Onn algorithm presented in the paper by Deza et al. [10] using the same generators. We provide the detailed numerical results of that paper in Table 2, taken from Huang's master thesis (in which the algorithm is referred to as $\mathrm{BO} 2)$.

\begin{tabular}{r|c|r|r|r|r|r|r|r|r|r} 
& \multicolumn{2}{|c|}{ Random } & \multicolumn{2}{c|}{ Tube } & \multicolumn{2}{c|}{ Highdensity } & \multicolumn{2}{|c}{ Lowdensity } & \multicolumn{2}{c}{ Middensity } \\
Dimension & time & \# pivots & time & \# pivots & time & \# pivots & time & \# pivots & time & \# pivots \\
\hline 3 & 0.7501 & 2.18 & 1.083 & 3.70 & 0.5735 & 1.53 & 0.9090 & 2.88 & 0.7613 & 2.24 \\
6 & 1.832 & 4.94 & 3.716 & 11.46 & 0.8450 & 1.73 & 2.404 & 6.81 & 1.936 \\
12 & 5.789 & 11.15 & 13.72 & 29.10 & 1.457 & 1.61 & 7.225 & 14.26 & 5.984 & 11.51 \\
24 & 21.88 & 24.94 & 56.23 & 69.98 & 3.685 & 1.65 & 24.91 & 28.53 & 21.38 & 23.76 \\
48 & 96.67 & 52.32 & 251.7 & 150.2 & 15.89 & 1.48 & 105.8 & 59.9 & 92.40 & 47.9 \\
96 & 513.1 & 107.9 & 1278 & 298.9 & 82.79 & 1.58 & 542.5 & 111.0 & 488.1 & 95.92 \\
192 & 3465 & 214.7 & 9253 & 625.8 & 559.7 & 1.8 & 3711 & 221.6 & 3274 & 191.9 \\
384 & 34230 & 433.6 & & & 4355 & 1.64 & 37010 & 459.8 & 30150 & 384
\end{tabular}

TABLE 2. Average solution time and number of pivots for the Bárány-Onn algorithm, as recorded in [14]

In general, our number of pivots is slightly larger than what they get. Regarding the computation time, it is hard to draw a conclusion since their implementation was done in 
MATLAB and since they used a different machine (a server with eight 64-bit CPUs, clocked at $2.6 \mathrm{GHz}$, with $64 \mathrm{~GB}$ RAM). However, we are always much faster - our total computation time is of the order of ten times smaller than theirs - and our time per iteration is up to thirty times smaller.

\section{Optimization in COLORFul LineAR PROGRAMming AND APPLiCATiOnS}

4.1. Optimization. The colorful linear programming problem is defined as a feasibility problem. However, it is natural to introduce an optimization version. For the usual linear programming problem, feasibility and optimization are equivalent: if we have a method for deciding whether a linear program is feasible, then we can use it for solving linear programs to optimality. The same holds for colorful linear programming, as we are now going to prove.

A colorful linear program is a mathematical program of the form

$$
\begin{array}{ll}
\min & \boldsymbol{c} \cdot \boldsymbol{x} \\
\text { s.t. } & A \boldsymbol{x}=\boldsymbol{b} \\
& \boldsymbol{x} \geq \mathbf{0} \\
& \left|\operatorname{supp}(\boldsymbol{x}) \cap I_{i}\right| \leq 1, \quad \forall i \in\{1, \ldots, k\}
\end{array}
$$

where $A$ is a $d \times n$ real matrix, $\boldsymbol{b}$ an element of $\mathbb{R}^{d}$, and $\boldsymbol{c}$ an element of $\mathbb{R}^{n}$, and where the $I_{i}$ 's form a partition of the columns of $A$, the number of colors being $k$. The problem of deciding whether a colorful linear program has a solution is exactly CoLORFul LinEAR Programming in its conic version.

Proposition 5. The two problems of deciding the feasibility of a colorful linear program and of finding the optimal solution of a colorful linear program are polynomially reducible to each other.

Proof. Clearly, the feasibility problem is reducible to the optimization problem. We show now the converse implication and let us assume that we can decide in polynomial time whether a colorful linear program has a feasible solution. We explain then how to compute an optimal solution of (Q).

We start by testing whether the problem (Q) has a feasible solution. If not, we are done. Otherwise, we compute a feasible solution by repeating several times the decision test: we apply it with the first color reduced to each point in turn, until the answer is 'yes', keep this point, and proceed similarly for all colors one after the other. It provides an upper bound $\mu_{\text {sup }}$ on the optimal value of (Q). We compute also a lower bound $\mu_{\text {inf }}$ by taking the noncolorful version of (Q), i.e., by relaxing the constraint

$$
\left|\operatorname{supp}(\boldsymbol{x}) \cap I_{i}\right| \leq 1 \quad \forall i
$$

This lower bound is polynomially computable since it is usual linear programming. 
Consider now the feasibility problem of deciding whether the following system has a solution. Using our polynomial decision test, we can solve it in polynomial time.

$$
\left\{\begin{array}{l}
\left(\begin{array}{ll}
A & 0 \\
\boldsymbol{c} & 1
\end{array}\right) \boldsymbol{x}^{\prime}=\left(\begin{array}{l}
\boldsymbol{b} \\
\mu
\end{array}\right) \\
\boldsymbol{x}^{\prime} \geq \mathbf{0} \\
\left|\operatorname{supp}\left(\boldsymbol{x}^{\prime}\right) \cap I_{i}\right| \leq 1 \\
\left|\operatorname{supp}\left(\boldsymbol{x}^{\prime}\right) \cap\{n+1\}\right| \leq 1 .
\end{array} \forall i\right.
$$

The colors for this new colorful linear program are the $I_{i}$ 's defined for the original program and an additional $(k+1)$ th color $I_{k+1}=\{n+1\}$. The problem (6) is feasible if and only if a colorful solution $\left(\begin{array}{l}\boldsymbol{x} \\ z\end{array}\right)$ of this problem induces a colorful solution $\boldsymbol{x}$ of (Q) with value smaller than $\mu$.

Using binary search, we can thus solve the problem. At each step, we consider the problem (6) with $\mu=\left(\mu_{\mathrm{inf}}+\mu_{\mathrm{sup}}\right) / 2$. If the problem is feasible, we update $\mu_{\text {sup }}:=\mu$, otherwise we update $\mu_{\text {inf }}:=\mu$. At each step the size of $\left[\mu_{\text {inf }}, \mu_{\text {sup }}\right]$ is hence divided by two. Using Cramer's rule, we know that there is a minimal gap $\varepsilon$, polynomially computable, between two values of $\boldsymbol{c} \cdot \boldsymbol{x}$ with $\boldsymbol{x}$ being a feasible basis of the system

$$
A \boldsymbol{x}=\boldsymbol{b}, \boldsymbol{x} \geq \mathbf{0} .
$$

Hence, after at most $\log _{2}\left(\frac{\mu_{\mathrm{sup}}-\mu_{\mathrm{inf}}}{\varepsilon}\right)$ iterations, the feasible basis we obtain for the problem with $\mu=\mu_{\text {sup }}$ is the optimum.

4.2. Colorful diet. Colorful linear programming can also be used as a modeling tool, as in the following example suggested to us by Max Klimm.

The diet problem has been introduced during the Second World War and aimed at defining the daily diet of U.S. soldiers. Given a set of nutriments and a set of foods, each containing a certain amount of each nutriment, the problem is to find an optimal diet with respect to some objective function, such that each nutriment is sufficiently provided. It was one of the first problems on which the simplex algorithm was tested (in 1947 [7]). Later, in 1990, Dantzig showed the limits of this model in an over-viewing paper [8], in which he described how he tried to apply the model to his own diet. The main struggle he encountered was "the lack of variety" of the solutions given by the model. Adding bounds, he managed to avoid solutions using only one food, for instance bran1. In this context, variety is thus achievable by standard linear programming technique. However, if he had known colorful linear programming, he would have had an additional modeling flexibility. It might be undesirable to have many foods from a same category in a solution, e.g., for logistic reasons. In general, one does not eat more than three types of vegetables, two types of meat and one fruit each day, and so on. Colorful linear programming consists in solving a linear program whose variables are partitioned into categories and with the additional constraints that the number of variables

\footnotetext{
${ }^{1}$ Dantzig finally followed his wife's advice, which was certainly much more efficient than linear programming.
} 
of each category used in a solution is bounded. It allows thus to avoid a too high variety inside the categories.

Formally, the original diet problem is the following. We are given $n$ foods and $m$ nutriments. Let $a_{i j} \in \mathbb{R}_{+}$be the quantity of nutriment $j$ in one unit of food $i$, let $b_{j}$ be the quantity of nutriment $j$ needed daily, and let $r_{i}$ be the maximal amount of food $i$ that can be tolerated in one day. Finally, let $c_{i}$ be the cost of one unit of food $i$. We define the variables $x_{1}, \ldots, x_{n} \in \mathbb{R}_{+}$, modeling the quantity of food $i$ that will be recommended by the diet program. The diet problem aims at solving

$$
\begin{array}{cl}
\min & \boldsymbol{c} \cdot \boldsymbol{x} \\
\mathrm{s.t.} & A \boldsymbol{x} \geq \boldsymbol{b} \\
& \boldsymbol{x} \leq \boldsymbol{r} \\
& \boldsymbol{x} \geq \mathbf{0},
\end{array}
$$

where $A$ is the matrix $\left(a_{i j}\right)$.

Now, assume that the foods are partitioned into different categories, i.e., $\{1, \ldots, n\}=$ $I_{1} \cup \cdots \cup I_{k}$, and that it is required to have at most $\ell_{h}$ distinct foods selected in a category $I_{h}$. This requirement can be taken into account by adding the following constraints to the previous linear program:

$$
\left|\operatorname{supp}(\boldsymbol{x}) \cap I_{h}\right| \leq \ell_{h} \quad \forall h \in\{1, \ldots, k\} .
$$

We get what we call the colorful diet problem.

By making $\ell_{h}$ copies of $I_{h}$ and by adding the corresponding $\left(x_{i, j}\right)_{j \in\left[\ell_{h}\right]}$ variables and by using slack variables, this model can be readily written under the form (Q). Proposition 5 shows that the colorful diet problem is polynomially reducible to Colorful LinEAR PROGRAMMING in its conic version.

\section{Complexity of COlORful linear Programming}

In this section, we come back to the original Colorful Linear Programming problem, discuss quickly its NP-completeness, and give an application.

5.1. Proof of NP-completeness. For a fixed $q \in \mathbb{Z}$, we define $\operatorname{CLP}(q)$ to Colorful Linear Programming with the additional constraint that $k-d=q$.

Lemma 3. If $\operatorname{CLP}(q)$ is $\mathrm{NP}$-complete, then $\operatorname{CLP}(q-1)$ is also $\mathrm{NP}$-complete.

Proof. Let $\mathbf{S}_{1}, \ldots, \mathbf{S}_{k}$ in $\mathbb{R}^{d}$ be an instance with $k=d+q$. Define $d^{\prime}=d+1$. Embedding this instance in $\mathbb{R}^{d^{\prime}}$ by adding a $d^{\prime}$ th component equal to 0 , we get an instance with $k=d^{\prime}+q-1$, every solution of which provides a solution for the case $k=d+q$, and conversely. This latter case being NP-complete, we get the conclusion.

Lemma 4. If $\operatorname{CLP}(q)$ is $\mathrm{NP}$-complete, then $\operatorname{CLP}(q+1)$ is also NP-complete.

Proof. Let $\mathbf{S}_{1}, \ldots, \mathbf{S}_{k}$ in $\mathbb{R}^{d}$ be an instance with $k=d+q$. Define $d^{\prime}=d+1$ and $k^{\prime}=k+2$. Embed this instance in $\mathbb{R}^{d^{\prime}}$ by adding a $d^{\prime}$ th component equal to 0 . Add two sets $\mathbf{S}_{k+1}$ and $\mathbf{S}_{k+2}$ entirely located at coordinate $(0, \ldots, 0,1)$. We have thus an instance with $k^{\prime}=d^{\prime}+q+1$, every solution of which provides a solution for the case $k=d+q$, and conversely. This latter case being NP-complete, we get the conclusion. 
We have the following proposition.

Proposition 6. $\operatorname{CLP}(q)$ is $\mathrm{NP}$-complete for any fixed $q \in \mathbb{Z}$.

In particular, it is NP-complete for $q=1$.

Proof of Proposition [6. CLP(0) is NP-complete according to Theorem 6.1 in [4]. Lemmas 3 and 4 allow to conclude.

Polynomially checkable sufficient conditions ensuring the existence of a positively dependent colorful set exist: the condition of the colorful Carathéodory theorem is one of them. More general polynomially checkable sufficient conditions when $k=d+1$ are given in [1, 12, 25. However, the fact that CLP(1) is NP-complete implies that there are no polynomially checkable conditions that are simultaneously sufficient and necessary for a positively dependent colorful set to exist when $k=d+1$, unless $\mathrm{P}=\mathrm{NP}$.

Remark 3. The instances built in the proof of Lemma 4 are not in general position, since $\mathbf{0}$ and the $\mathbf{S}_{i}$ 's with $i \leq k$ are all in a same hyperplane. We could wonder whether the case $k=d+1$ remains NP-complete when the points are in general position. The answer is yes, and we explain how to reduce the instance built in the proof of Lemma 4 to an instance in general position.

First, the sets $\mathbf{S}_{k+1}$ and $\mathbf{S}_{k+2}$ can be slightly perturbed without changing the conclusion. Second, we slightly move $\mathbf{0}$ into one of the halfspaces delimited by the hyperplane containing the $\mathbf{S}_{i}$ 's for $i \leq k$. We choose the halfspace containing $\mathbf{S}_{k+1}$ and $\mathbf{S}_{k+2}$. This move must be sufficiently small so that $\mathbf{0}$ does not traverse another hyperplane generated by $d^{\prime}$ points in $\bigcup_{i=1}^{k+2} \mathbf{S}_{i}$. All coordinates being rational, Cramer's formula allows to compute a length of the displacement that ensures this condition. Third, we move each point of the $\bigcup_{i=1}^{k} \mathbf{S}_{i}$ independently along a line originating from $\mathbf{0 .}$

5.2. Projection and colorful linear programming. Algorithmic questions related to projecting polytopes are usually identified as difficult questions. Tiwary [33] recently showed that given two polytopes $\mathcal{Q}$ and $\mathcal{Q}^{\prime}$ described by systems of linear inequalities, deciding whether $\mathcal{Q}$ is a projection of $\mathcal{Q}^{\prime}$ is coNP-complete. Note that it is in coNP since deciding whether a partial solution of a system of linear inequalities can be extended to a full solution is a linear programming problem. His proof of coNP-completeness uses a reduction of the problem of deciding whether a polytope described by its facets is contained in a polytope described by its vertices, which is a coNP-complete problem [11]. Colorful Linear Programming is another way to prove this result.

Take any instance $\mathbf{S}_{1}, \ldots, \mathbf{S}_{d}, \boldsymbol{p}$ of the conic version of Colorful Linear ProgramMING, all points being in general position, with $\operatorname{conv}\left(\{\boldsymbol{p}\} \cup \bigcup_{i=1}^{d} \mathbf{S}_{i}\right)$ not containing $\mathbf{0}$. Because of Proposition 6 and Remark 3, the problem of deciding whether there is colorful solution is NP-complete. We show how to reduce this instance to an instance of the aforementioned polytope projection problem. We define $A_{i}$ to be the matrix with the columns being the vectors in $\mathbf{S}_{i}$, for $i=1, \ldots, d$. We define then the following polytopes:

$$
\mathcal{P}=\left\{\boldsymbol{x}=\left(\boldsymbol{x}_{1}, \ldots, \boldsymbol{x}_{d}\right) \in \mathbb{R}_{+}^{d^{2}}: \sum_{i=1}^{d} A_{i} \boldsymbol{x}_{i}=\boldsymbol{p}\right\}
$$


and

$$
\mathcal{P}_{i}=\left\{\boldsymbol{x}=\left(\boldsymbol{x}_{1}, \ldots, \boldsymbol{x}_{d}\right) \in \mathcal{P}: \boldsymbol{x}_{i}=\mathbf{0}\right\} .
$$

They are polytopes because of the assumption $\operatorname{conv}\left(\{\boldsymbol{p}\} \cup \bigcup_{i=1}^{d} \mathbf{S}_{i}\right)$ does not contain $\mathbf{0}$.

There exists a colorful solution to COLORful Linear PROGRAMMing problem we consider if and only if $\mathcal{P} \backslash \operatorname{conv}\left(\bigcup_{i=1}^{d} \mathcal{P}_{i}\right)$ is nonempty. Indeed, if there exists a colorful solution to the Colorful Linear Programming problem, the latter set in nonempty: a colorful solution provides a point (a basis in the linear programming terminology) in $\mathcal{P}$ with each $\boldsymbol{x}_{i}$ being nonzero, because of the general position assumption. Conversely, if the set is nonempty, there is a vertex of $\mathcal{P}$ not in $\operatorname{conv}\left(\bigcup_{i=1}^{d} \mathcal{P}_{i}\right)$, and such a vertex has exactly $d$ nonzero components, each corresponding to a column of a distinct $A_{i}$, and provides a solution to the Colorful Linear Programming problem we consider.

Deciding whether $\mathcal{P} \backslash \operatorname{conv}\left(\bigcup_{i=1}^{d} \mathcal{P}_{i}\right)$ is nonempty is therefore NP-hard. We prove below that $\operatorname{conv}\left(\bigcup_{i=1}^{d} \mathcal{P}_{i}\right)$ is a projection of some higher dimensional polytope $\mathcal{Q}^{\prime}$. Hence, deciding whether $\mathcal{P} \backslash \operatorname{conv}\left(\bigcup_{i=1}^{d} \mathcal{P}_{i}\right)$ is nonempty is equivalent to deciding whether $\mathcal{P}$ is the projection of $\mathcal{Q}^{\prime}$.

The polytope conv $\left(\bigcup_{i=1}^{d} \mathcal{P}_{i}\right)$ is described by the solutions $\boldsymbol{x}=\left(\boldsymbol{x}_{1}, \ldots, \boldsymbol{x}_{d}\right)$ satisfying the following system of linear equalities and inequalities:

$$
\begin{cases}\sum_{j=1}^{d} A_{i} \boldsymbol{x}_{i j}-y_{i} \boldsymbol{p}=\mathbf{0} & \forall i \\ \sum_{i=1}^{d} \boldsymbol{x}_{i j}=\boldsymbol{x}_{j} & \forall j \\ \sum_{i=1}^{d} y_{i}=1 & \\ \boldsymbol{x}_{i i}=\mathbf{0} & \forall i \\ \boldsymbol{y} \geq \mathbf{0} & \forall i \\ \boldsymbol{x}_{i j} \geq \mathbf{0} & \forall i, j .\end{cases}
$$

Indeed, a point $\boldsymbol{x}=\left(\boldsymbol{x}_{1}, \ldots, \boldsymbol{x}_{d}\right) \in \operatorname{conv}\left(\bigcup_{i=1}^{d} \mathcal{P}_{i}\right)$ is such that $\boldsymbol{x}=\sum_{i=1}^{d} y_{i} \boldsymbol{x}_{i}^{\prime}$, with $\sum_{i=1}^{d} y_{i}=1$ and $\boldsymbol{x}_{i}^{\prime}=\left(\boldsymbol{x}_{i 1}^{\prime}, \ldots, \boldsymbol{x}_{i d}^{\prime}\right) \in \mathcal{P}_{i}$ for each $i$. Defining $\boldsymbol{x}_{i j}$ to be $y_{i} \boldsymbol{x}_{i j}^{\prime}$ shows that such an $\boldsymbol{x}$ satisfies the system. Conversely, a solution of the system induces a point $\boldsymbol{x}$ that can be written as $\sum_{i=1}^{d} y_{i} \boldsymbol{x}_{i}^{\prime}$ with $\boldsymbol{x}_{i}^{\prime} \in \mathcal{P}_{i}$ for all $i$. Indeed, define $\boldsymbol{x}_{i j}^{\prime}=\frac{1}{y_{i}} \boldsymbol{x}_{i j}$ when $y_{i} \neq 0$, and $\boldsymbol{x}_{i j}^{\prime}=\mathbf{0}$ otherwise. In this latter case, all the $\boldsymbol{x}_{i j}$ 's are equal to $\mathbf{0}$ because of the assumption $\mathbf{0} \notin \operatorname{conv}\left(\{\boldsymbol{p}\} \cup \bigcup_{i=1}^{d+1} \boldsymbol{S}_{i}\right)$.

\section{Special Cases and analogues of COlorful linear programming in COMBINATORICS}

We give in this section two combinatorial corollaries of the colorful Carathéodory theorem. For each of them, we provide a direct proof. In both cases, we show that the colorful set can be computed in polynomial time, and get thus special polynomial cases of ColorfuL CARATHÉODORY. 
Proposition 7. Let $D=(V, A)$ be a directed graph with $n$ vertices. Let $C_{1}, \ldots, C_{n}$ be pairwise arc-disjoint circuits. Then there exists a circuit $C$ sharing at most one arc with each of these $C_{i}$. Moreover, such a circuit can be computed in polynomial time.

The existence of the colorful circuit as a consequence of the colorful Carathéodory theorem has already been noted and is attributed to Frank and Lovász [2].

Proof. We consider the bipartite graph with vertex classes $V$ and $\{1, \ldots, n\}$, and in which edge $v i$ exists if the vertex $v$ belongs to $C_{i}$. If each $X \subseteq V$ touches at least $|X|$ distinct colors, Hall's marriage theorem ensures the existence of a perfect matching in the bipartite graph. We can thus select for each vertex $v \in V$ an $\operatorname{arc} a$ in $\delta^{-}(v)$ belonging to a distinct $C_{i}$. The subgraph induced by these $\operatorname{arcs}$ contains a circuit $C$ as required.

Otherwise, there is a subset $X \subseteq V$ with a neighborhood in the bipartite graph of cardinality at most $|X|-1$. One can remove $X$ from $D$ and apply induction. Note that the existence of such an $X$ can be decided in polynomial time by a classical maximum matching algorithm, which provides also the set $X$ itself if it exists.

The existence statement of the next proposition is a consequence of the conic version of the colorful Carathéodory theorem. We provide a direct proof based on a greedy algorithm.

Proposition 8. Let $D=(V, A)$ be a directed graph with $n$ vertices. Let $s$ and $t$ be two vertices, and $P_{1}, \ldots, P_{n-1}$ be pairwise arc-disjoint s-t paths. Then there exists an $s$-t path $P$ sharing at most one arc with each $P_{i}$. Moreover, such a path can be computed in polynomial time.

Proof. We build progressively an arborescence rooted at $s$. We start with $X=\{s\}$. At each step, $X$ is the set of vertices reachable from $s$ in the partial arborescence. At step $i$, if $X$ does not contain $t$, choose an arc $a$ of $P_{i}$ belonging to $\delta^{+}(X)$ and add to $X$ the endpoint of $a$ not yet in $X$. This arc exists since by direct induction $X$ is of cardinality $i$ at step $i$ and the $s$ - $t$ path $P_{i}$ leaves $X$.

We end the paper by a brief survey of matroidal counterparts of colorful linear programming. The next proposition is common knowledge in combinatorics. It is a matroidal version of the colorful Carathéodory theorem (with an additional algorithmic result).

Proposition 9. Let $M$ be a matroid of rank d. Assume that the elements of $M$ are colored in d colors. If there exists a monochromatic basis in each color, then there exists a colorful basis and this latter can be found by a greedy algorithm.

A matroidal version of the Octahedron lemma stated in Section 2.1 also exists. It is due to Magnanti 21].

Proposition 10. Let $M$ be a matroid of rank d with no loops. Assume that the elements of $M$ are colored in d colors and that the number of elements colored in each color is at least two. If there is a colorful basis, then there is another colorful basis and this latter can then be found in polynomial time.

The proof by Magnanti is based on the matroid intersection algorithm due to Lawler [19]. The same algorithm shows that the matroidal version of Colorful Linear ProgramMING, namely deciding whether there is a colorful basis in a matroid whose elements are colored, is polynomial. 
Remark 4 (Back to Sperner's lemma). Remark 2 of Section 2.3 shows that even a very special case of Sperner's lemma already leads to a PPAD-complete problem. The matroidal counterpart of the Octahedron lemma implies that the problem becomes polynomial when the triangulation is the boundary of the cross-polytope. The cross-polytope is the convex hull of the vectors of the standard orthonormal basis and their negatives.

Proposition 11. Let $\mathrm{T}$ be the boundary of the $(d+1)$-dimensional cross-polytope and let $\lambda: V(\mathrm{~T}) \rightarrow\{1, \ldots, d+1\}$ be any labeling. Assume given a fully-labeled simplex. Another fully-labeled simplex can be computed in polynomial time.

Proof. If a vertex has a label that appears only once on $V(\mathrm{~T})$, we remove it and its antipodal, and work on the boundary of a cross-polytope with a dimension smaller by one. Solving this new problem leads to a solution for the original problem. We repeat this process until each label appears exactly twice. Now, note that the simplices of the boundary of a cross-polytope form the independent sets of a matroid (it is a partition matroid). Considering the labels as colors, the conclusion follows then from Proposition 10.

With a similar proof (omitted), we also have the following proposition.

Proposition 12. Let $\mathrm{T}$ be the boundary of the $(d+1)$-dimensional cross-polytope and let $\lambda: V(\mathrm{~T}) \rightarrow\{1, \ldots, d+1\}$ be any labeling. Deciding whether there is a fully-labeled simplex can be done in polynomial time. Moreover, if there is such a fully-labeled simplex, it can be found in polynomial time as well.

Acknowledgments. The authors thank the reviewers for their helpful comments.

\section{REFERENCES}

1. J. L. Arocha, I. Bárány, J. Bracho, R. Fabila, and L. Montejano, Very colorful theorems, Discrete and Computational Geometry 42 (2009), 142-154.

2. I Bárány, A generalization of Carathéodory's theorem, Discrete Mathematics 40 (1982), 141-152.

3. I. Bárány and J. Matoušek, Quadratically many colorful simplices, SIAM Journal on Discrete Mathematics 21 (2007), 191-198.

4. I. Bárány and S. Onn, Colourful linear programming and its relatives, Mathematics of Operations Research 22 (1997), 550-567.

5. X. Chen and X. Deng, On the complexity of $2 D$ discrete fixed point problem, Automata, Languages and Programming, Lecture Notes in Computer Science, vol. 4051, Springer Berlin Heidelberg, 2006, pp. $489-500$.

6. X. Chen, X. Deng, and S.-H. Teng, Settling the complexity of computing two-player Nash equilibria, Journal of the ACM 56 (2009), 14:1-14:57.

7. G. B. Dantzig, Linear programming and extensions, Princeton University Press, August 1963.

8. — The diet problem, Interfaces 20 (1990), no. 4, 43-47.

9. A. Deza, S. Huang, T. Stephen, and T. Terlaky, Colourful simplicial depth, Discrete and Computational Geometry 35 (2006), 597-604.

10. _ The colourful feasibility problem, Discrete Applied Mathematics 156 (2008), 2166-2177.

11. Robert M. Freund and James B. Orlin, On the complexity of four polyhedral set containment problem, Mathematical Programming 33 (1985), 139-145.

12. A. F. Holmsen, J. Pach, and H. Tverberg, Points surrounding the origin, Combinatorica 28 (2008), 633-644. 
13. S. Huang, MATLAB code for Colourful Linear Programming, available at: http://optlab.mcmaster.ca/ huangs3/CLP and http://www.math.sfu.ca/ tamon/Software/CLP, 2005.

14. _ Colourful feasibility: algorithms, bounds and implications, Master's thesis, Graduate Studies, McMaster University, 2007.

15. D. S. Johnson, C. H. Papadimitriou, and M. Yannakakis, How easy is Local Search, Journal of Computer and System Sciences 37 (1988), 79-100.

16. S. Kintali, L. J. Poplawski, R. Rajaraman, R. Sundaram, and S.-H. Teng, Reducibility among fractional stability problems, SIAM Journal on Computing 42 (2013), 2063-2113.

17. T. Király and J. Pap, PPAD-completeness of polyhedral versions of Sperner's Lemma, Discrete Mathematics 313 (2013), 1594-1599.

18. T. Kitahara and S. Mizuno, An upper bound for the number of different solutions generated by the primal simplex method with any selection rule entering variables, Asia-Pacific Journal of Operational Research 30 (2013).

19. E. L. Lawler, Matroid intersection algorithms, Mathematical programming 9 (1975), 31-56.

20. C. E. Lemke and J. T. Howson, Equilibrium points of bimatrix games, Journal of the Society for Industrial and Applied Mathematics 12 (1964), 413-423.

21. T. L. Magnanti, Complementary bases of a matroid, Discrete Mathematics 8 (1974), 355-361.

22. J. Matoušek, Lectures on discrete geometry, Springer, 2002.

23. N. Megiddo and R. Chandrasekaran, On the $\varepsilon$-perturbation method for avoiding degeneracy, Operations Research Letters 8 (1989), 305-308.

24. F. Meunier, Computing and proving with pivots, RAIRO - Operations Research 47 (2013), 331-360.

25. F. Meunier and A. Deza, A further generalization of the colourful Carathéodory theorem, Discrete Geometry and Optimization (Karoly Bezdek, Antoine Deza, and Yinyu Ye, eds.), Fields Institute Communications, vol. 69, Springer International Publishing, 2013, pp. 179-190.

26. W. Mülzer and Y. Stein, Complexity of finding nearest colorful polytopes, Proceedings of EuroCG, March 2014.

27. C. H. Papadimitriou, On the complexity of the parity argument and other inefficient proofs of existence, Journal of Computer and System Sciences 48 (1994), 498-532.

28. R. Rado, A theorem on independence relations, The Quaterly Journal of Mathematics (Oxford) 13 (1942), 83-89.

29. H. Scarf, The approximation of fixed points of a continuous mapping, SIAM Journal on Applied Mathematics 15 (1967), 1328-1343.

30. Alexandrer Schrijver, Theory of linear and integer programming, Wiley, 1986.

31. _ Combinatorial optimization, Springer, 2003.

32. E. Sperner, Neuer Beweis für die Invarianz der Dimensionszahl und des Gebietes, Abhandlungen aus dem Mathematischen Seminar der Universität Hambourg 6 (1928), 265-272.

33. Hans R. Tiwary, Complexity of computing the projection of polytopes, Tech. report, 2012.

34. M. J. Todd, Orientation in complementary pivot algorithms, Mathematics of Operations Research $\mathbf{1}$ (1976), 54-66.

35. L. A. Végh and B. von Stengel, Oriented Euler complexes and signed perfect matchings, Mathematical Programming 150 (2015), 153-178.

E-mail address: frederic.meunier@enpc.fr

E-mail address: pauline.sarrabezolles@enpc.fr 